\title{
Jim Connolly
}

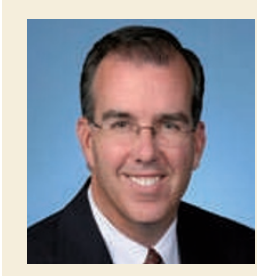

President and Chief Executive Officer, Aeras Global TB Vaccine Foundation, Maryland, USA. Prior to joining Aeras, Jim Connolly spent 23 years at Wyeth. Over this time, he held a number of increasingly senior positions ranging from finance to general management, and from 2005 to 2009 he was Executive Vice President and General Manager of Wyeth Vaccines. He played a leading role in growing Wyeth's vaccines business to a value of over US\$3 billion annually.

What attracted you to become President and Chief Executive Officer of the Aeras Global TB Vaccine Foundation?

For the last 4 years that I spent at Wyeth, I oversaw the vaccines business and I am very proud of what we accomplished. For example, Prevnar (a vaccine that prevents invasive disease caused by Streptococcus pneumoniae), which became the first true blockbuster vaccine, was the first product to demonstrate that it is possible to develop a vaccine that can have a dramatic impact on public health, as well as to provide significant financial returns for a company. Part of my role involved trying to expand access to Prevnar, particularly in developing countries. This culminated in the launch of Prevnar in the Gambia and Rwanda, and our participation in the Pneumococcal Advance Market Commitment, whereby the company committed to make Prevnar and the next-generation version, Prevnar 13, widely available for use in developing countries.

My work at Wyeth Vaccines whetted my appetite for the vaccine field, which was one of the main reasons why I was attracted to the Aeras Global TB Vaccine Foundation. From a societal perspective, this is also an opportunity to have an impact on tuberculosis (TB), a disease that kills nearly 2 million people each year. Another factor is that Aeras is an organization that has broad-based capabilities that range from basic research and development (R\&D) through to clinical development and manufacturing, as well as a talented, dedicated team of people to drive the development of a diverse portfolio of potential new products and technologies.

What are the main lessons that you learned when you were leading Wyeth Vaccines that could be applied to help facilitate the rapid development of TB vaccines?

At Wyeth Vaccines, we developed an enhanced business operating model that strongly integrated the operations and activities across the commercial, R\&D and manufacturing organizations. This allowed us to position Wyeth Vaccines with a much stronger commercial platform and pipeline, and created the opportunity to launch a series of new products over the coming years. My ability to build and leverage strong stakeholder relationships, in combination with my experience of integrating the diverse capabilities at Wyeth, is directly relevant to Aeras, which undertakes a full range of activities including in-house R\&D and manufacturing, and interactions with dozens of global partners. Another lesson that I have learned at Wyeth is the crucial need and importance of increasing disease awareness, which we need to do globally for TB to achieve our goals.

What are the main challenges for developing $T B$ vaccines and how are they being addressed by the Foundation?

The main challenges are to prevent Mycobacterium tuberculosis infection and to prevent re-activation of latent TB. The TB vaccine bacille Calmette-Guérin (BCG), which was invented nearly 90 years ago, is widely available and used. However, it has had little impact on the growing TB epidemic because it is unreliable at preventing pulmonary TB infection, which accounts for most of the TB disease worldwide, and does not seem to protect against latent TB. In addition, it is not recommended for use in infants infected with HIV.

To address these challenges, Aeras is pursuing work in three distinct areas. First, our scientists have shown that a recombinant BCG candidate that overexpresses key antigens in a manner more like $M$. tuberculosis has the potential to prime the immune system better than the existing BCG vaccine [which is based on an attenuated mycobacterial strain], and we hope to move that into clinical trials in the near future. Second, we are pursuing a prime-boost scenario using the current $B C G$ vaccine or a recombinant BCG vaccine coupled with a booster vaccine. This could enhance the protection that is afforded by the BCG vaccine, as well as extend protection over a longer period of time. Third, we are working with thought leaders and experts around the world to look at new approaches, technologies and delivery systems that were not available until recent years and to harness these advances to effectively deliver TB vaccines.

What kinds of partnerships has Aeras established to develop TB vaccines? And what is the main challenge for bringing these vaccines to the clinic?

Aeras is a non-profit product development partnership that is working with a range of partners in different areas. These include the academic area, such as Oxford University, UK; quasi-governmental organizations, such as the Statens Serum Institute of Denmark; biotechnology companies, such as Crucell and Intercell; and some of the larger global pharmaceutical companies, such as GlaxoSmithKline and Sanofi-Pasteur. We have also created a network of clinical trial partners in Africa and Asia who are also crucial players in accomplishing our mission. A successful vaccine will not come from one entity working by itself. It will only occur by harnessing the capabilities, the passion, the resources and the innovations that exist across numerous partners around the world. This was why Aeras was created - to help make the development of new vaccines against TB a reality.

One of the biggest challenges today and it is exacerbated by the current economic downturn - is mobilizing the resources to fund our work, particularly when we want to start larger and later-stage trials. We have been highly fortunate to receive funding from various governments and donors like the Bill \& Melinda Gates Foundation, but vaccine $\mathrm{R} \& \mathrm{D}$, even in a non-profit setting, requires tens if not hundreds of millions of dollars of investment. As a result, we are going to need to sustain the level of funding, and also to increase and expand our donor and funding base in order to achieve our goal of reducing and eliminating the global burden of TB. 